ARTICLE

Received 17 Oct 2014 | Accepted 5 May 2015 | Published 22 Jun $2015 \quad$ DOl: 10.1038/ncomms8395

\title{
Peptidyl-prolyl isomerization targets rice Aux/IAAs for proteasomal degradation during auxin signalling
}

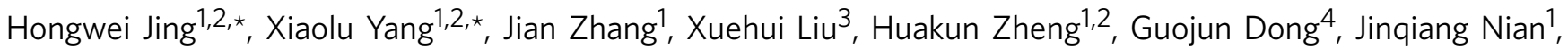
Jian Feng ${ }^{1}$, Bin Xia ${ }^{5}$, Qian Qian ${ }^{4}$, Jiayang $\mathrm{Li}^{1} \&$ Jianru Zuo ${ }^{1}$

In plants, auxin signalling is initiated by the auxin-promoted interaction between the auxin receptor TIR1, an E3 ubiquitin ligase, and the Aux/IAA transcriptional repressors, which are subsequently degraded by the proteasome. Gain-of-function mutations in the highly conserved domain II of Aux/IAAs abolish the TIR1-Aux/IAA interaction and thus cause an auxin-resistant phenotype. Here we show that peptidyl-prolyl isomerization of rice OsIAA11 catalysed by LATERAL ROOTLESS2 (LRT2), a cyclophilin-type peptidyl-prolyl cis/trans isomerase, directly regulates the stability of OsIAA11. NMR spectroscopy reveals that LRT2 efficiently catalyses the cis/trans isomerization of OsIAA11. The Irt2 mutation reduces OsTIR1-OsIAA11 interaction and consequently causes the accumulation of a higher level of OsIAA11 protein. Moreover, knockdown of the OsIAA11 expression partially rescues the Irt2 mutant phenotype in lateral root development. Together, these results illustrate cyclophilincatalysed peptidyl-prolyl isomerization promotes Aux/IAA degradation, as a mechanism regulating auxin signalling.

\footnotetext{
${ }^{1}$ State Key Laboratory of Plant Genomics and National Center for Plant Gene Research, Institute of Genetics and Developmental Biology, Chinese Academy of Sciences, Beijing 100101, China. ${ }^{2}$ University of Chinese Academy of Sciences, Beijing 100049, China. ${ }^{3}$ Institute of Biophysics, Chinese Academy of Sciences, Beijing 100101, China. ${ }^{4}$ State Key Laboratory of Rice Biology, China National Rice Research Institute, Chinese Academy of Agricultural Sciences, Hangzhou 310006, China. ${ }^{5}$ College of Life Sciences, Peking University, Beijing 100871, China. * These authors contributed equally to this work. Correspondence and requests for materials should be addressed to J.Z. (email: jrzuo@genetics.ac.cn).
} 
A uxin is an essential phytohormone that plays a fundamental role in the regulation of nearly all aspects of plant growth and development. In the past decades, extensive genetic and biochemical studies in Arabidopsis reveal that auxin signalling is mediated by a cascade that regulates the proteasomal degradation of the Auxin/INDOLE ACETIC ACID (Aux/IAA) transcriptional repressors in an auxin-dependent manner ${ }^{1-3}$. When the intracellular concentration of auxin is low, Aux/IAAs directly interact with AUXIN RESPONSE FACTORS (ARFs), a class of transcription factors that control the expression of downstream auxin-responsive genes, and thereby inhibit the transcriptional activity of ARFs. In response to specific developmental or environmental cues, auxin promotes the formation of a co-receptor complex consisting of a ubiquitin-ligase SCF ${ }^{\text {TIR1/AFB }}$ and Aux/IAAs (refs 4-6). The auxin-dependent formation of the $\mathrm{SCF}^{\mathrm{TIR} 1}$-Aux/IAA complex results in the ubiquitination of Aux/IAAs, which are subsequently subjected to degradation through the $26 \mathrm{~S}$ proteasomal degradation machinery. The proteasomal destruction of Aux/ IAAs relieves ARFs from the repressive complex and the active ARFs directly bind to the promoters to activate or repress the transcription of the downstream target genes ${ }^{7-11}$.

The Aux/IAA repressor proteins contain four highly conserved domains, named domains I-IV (ref. 10). Of those domains, domain II, termed as the degron motif, is a key determinant of auxin-dependent degradation of Aux/IAAs by mediating interaction with the auxin receptors TRANSPORT INHIBITOR RESPONSE1/AUXIN SIGNALING F-BOX PROTEINS (TIR1/ AFBs). Genetic studies reveal that various gain-of-function mutations in domain II, largely located in the highly conserved central Gly-Trp-Pro-Pro-Val (GWPPV) motif, render Aux/ IAAs resistant to the auxin-induced proteasomal degradation, resulting in a dominant auxin insensitive phenotype $e^{7-10,12-14}$. Notably, the auxin-dependent interaction between TIR1 and Aux/IAAs is reduced or abolished by mutations in the GWPPV motif, illustrating the critical role of the domain II-mediated degradation of Aux/IAAs in auxin signalling $4,5,7,13,15$. Consistent with these observations, the analysis of the crystal structure of the Arabidopsis TIR1-auxin-IAA7 peptide complex reveals that the GWPPV motif of domain II is crucial for the formation of the coreceptor complex ${ }^{6}$. These studies demonstrate that the domain IImediated degradation of Aux/IAAs is a key step in auxin signalling. However, the possible involvement of posttranslational modifications of Aux/IAAs in regulating their stability remains elusive.

Recent studies suggest that rice, a model species of monocotyledonous plants, employs a regulatory mechanism of auxin signalling similar to that in Arabidopsis. Mutations in the rice LATERAL ROOTLESS2 (LRT2; also known as Oryza sativa CYCLOPHILIN2 or OsCYP2) gene cause an auxin-resistant phenotype and defective development of lateral roots ${ }^{16,17}$. LRT2 encodes a putative cyclophilin-type peptidyl-prolyl cis/trans isomerase (PPIase) that catalyses the isomerization of peptide bonds at proline residues to specifically regulate protein conformational changes. The PPIase superfamily in higher eukaryotes includes cyclophilins (CYP), FK506-binding proteins, parvulins and PP2A phosphatase activators ${ }^{18,19}$. In plants, cyclophilin-like genes have been identified from a variety of species, including both Arabidopsis and rice ${ }^{20-22}$. A number of plant CYP genes, mainly in Arabidopsis, have been functionally characterized, involved in a variety of physiological and developmental processes, including in flowering, phytohormone signalling, stress responses and immune responses ${ }^{22-27}$. Mutations in LRT2-like genes of the tomato DIAGEOTROPICA (DGT) and the moss Physcomitrella patens PpDGT genes also caused an auxin-resistant phenotype ${ }^{28-30}$, indicating that this class of highly conserved genes plays an important role in regulating auxin signalling in both lower and higher plants. However, how the cyclophilin-type PPIases function in auxin signalling remains unknown. Here we show that rice LRT2/ OsCYP2 acts as a functional cyclophilin to catalyse the peptidylprolyl cis/trans isomerization of the OsAux/IAA transcriptional repressors, thereby facilitating their binding to OsTIR1 for subsequent proteasomal degradation and eventually activating auxin signalling.

\section{Results}

LRT2 physically interacts with OsAux/IAA proteins. Previous studies showed that mutations in the rice LRT2/OsCYP2 gene (referred to as LRT2 hereafter) cause an auxin-resistant phenotype and defective development of lateral roots ${ }^{16,17}$. LRT2 encodes a putative cyclophilin-type PPIase that catalyses the isomerization of peptide bonds at Pro residues. However, the specific substrates of LRT2 remain unknown. We noticed that the lrt 2 mutant phenotype was similar to that of the rice osiaa11, osiaa13 and osiaa23 mutants $^{31-33}$ (Supplementary Fig. 1). In particular, mutations in LRT2, OsIAA11, OsIAA13 and OsIAA23 all cause defective lateral root primordia, reduced tillering and plant height, and impaired panicle development, accompanying with the reduced expression level of auxininducible genes and the altered expression pattern of the DR5:GUS reporter gene ${ }^{16,17,31-33}$, suggesting that LRT2 may directly target OsAux/IAAs.

A close examination of the known mutations in domain II in various species revealed that these mutations occurred mainly in the two absolutely conserved Pro residues or, in a few cases, in the adjacent residues (Supplementary Fig. 2), suggesting that these two Pro residues play a critical role in regulating the stability of Aux/IAA proteins ${ }^{10,15}$. If LRT2 indeed targets OsAux/IAAs, these two types of proteins should directly interact. In a luciferase complementation imaging (LCI) assay, we found that LRT2 interacted with all the tested OsAux/IAA proteins with various affinities when transiently expressed in tobacco leaves (Fig. 1a; Supplementary Fig. 3). Similarly, LRT2 interacted with OsIAA11 and OsIAA13 in a yeast two-hybrid assay (Fig. 1b). A protein pull-down experiment revealed that LRT2 directly interacted with OsIAA11 and OsIAA13 proteins (Fig. 1c). Moreover, LRT2 interacted with OsIAA11 and OsIAA13 proteins in planta as revealed by a co-immunoprecipitation (Co-IP) assay (Fig. 1d; Supplementary Fig. 4). In the Co-IP experiment, we reproducibly observed an extra band with the reduced size in the osiaa11 sample by using two batches of antibodies generated from different mice. We speculate that this band may be a degraded product of osiaa11 mutant protein in the assay. Notably, the osiaa11 (OsIAA11 ${ }^{\mathrm{P} 106 \mathrm{~L}}$ ) gain-of-function mutant protein showed the reduced interaction with LRT2 compared with its wild-type partners (Fig. 1d). When transiently expressed in tobacco leaves, the direct interactions between LRT2 and OsIAA11 or OsIAA13 were also detected by a Co-IP assay (Supplementary Fig. 5). Moreover, mutations in the conserved Pro residues

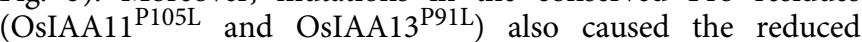
interactions with LRT2, suggestive of the importance of these Pro residues in mediating the LRT2-OsAux/IAA interaction. Taken together, these results demonstrated that LRT2 directly interacts with OsIAA11 and OsIAA13 and the interaction is regulated by domain II of OsAux/IAA proteins.

LRT2 catalyses cis/trans isomerization of OsIAA11 peptide. Given that LRT2 physically interacts with OsIAA11 and OsIAA13, we next explored whether LRT2 is capable of catalysing the peptidyl-prolyl isomerization of OsAux/IAA proteins by 
a

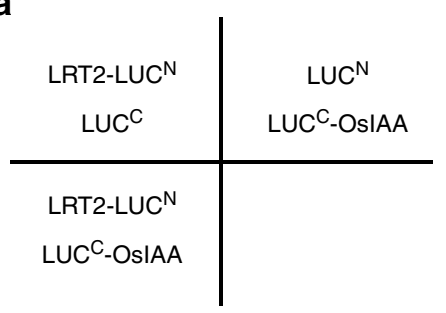

b

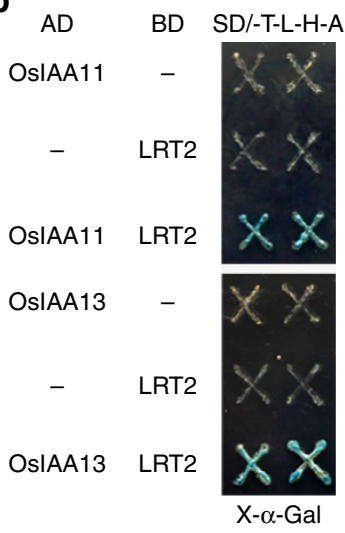

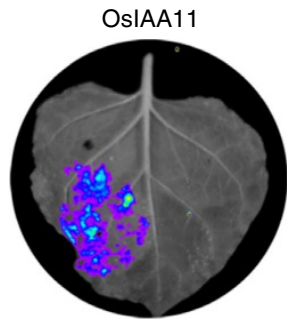

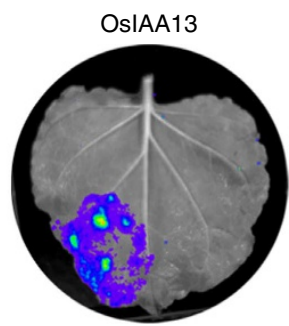

C

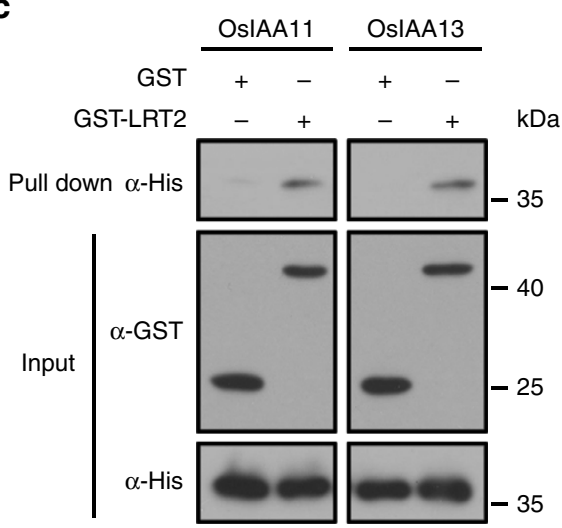

d
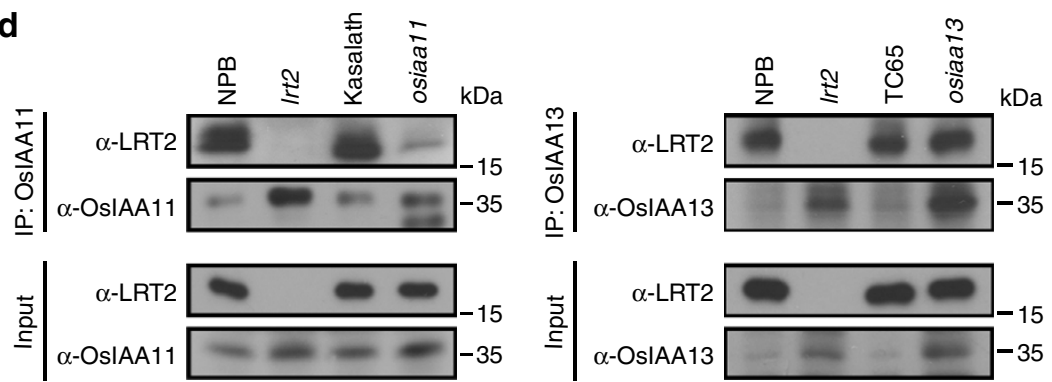

Figure 1 | LRT2 interacts with OsIAA proteins. (a) Analysis of the interaction of LRT2 with OsIAA11 and OsIAA13 transiently expressed in tobacco leaves by the luciferase complementation imaging assay. (b) Analysis of the interaction of LRT2 with OsIAA11 and OsIAA13 by the yeast two-hybrid assay. (c) GST-LRT2 directly interacts with His-OsIAA11 (OsIAA11) and His-OsIAA13 (OsIAA13) as analysed by the pull-down experiment. GST protein is served as a negative control. (d) Co-immunoprecipitation analysis of the interaction of LRT2 with OsIAA11 and OsIAA13 in wild-type (Nipponbare or NPB, Kasalath and Taichung 65 or TC65), Irt2, osiaa11 and osiaa13 seedlings.

NMR spectroscopy. To this end, we examined the capability of LRT2 for catalysing cis/trans conformational exchange of a peptide of 12 residues derived from domain II of OsIAA11 (residues 98-109), which differed in one residue with OsIAA13 and Arabidopsis IAA7 in this region (Fig. 2a; Supplementary Fig. 2). Analysis of the crystal structure of the TIR1-IAA7 co-receptor revealed that $\operatorname{Trp}^{86}$ and $\operatorname{Pro}^{88}$ in the conserved central GWPPV motif of the IAA7 peptide are inserted in the auxin-binding pocket of TIR1 and stacking against the auxin indole ring and the auxin side chain, respectively, whereas Pro ${ }^{87}$ in cis-conformation is important for maintaining the binding conformation ${ }^{6}$.

We found that LRT2 efficiently catalysed cis/trans conformational exchanges of the OsIAA11 peptide as evident by the detection of exchange peaks resulting from proline isomerization in the ROESY (rotating frame Overhauser effect spectroscopy) spectra, whereas no exchange peak was detected in the absence of LRT2 (Fig. 2b,c), suggesting that LRT2 is capable of catalysing conformational exchanges of the OsIAA11 peptide. We also analysed the PPIase activity of LRT2 ${ }^{\mathrm{G} 72 \mathrm{~A}}$ mutant protein. $\mathrm{Gly}^{72}$ is a highly conserved residue located in the PPIase domain (Supplementary Fig. 6a) and mutations in this residue of rice LRT2/OsCYP2 and moss PpDGT cause severely impaired auxin signalling ${ }^{17,30}$. Homologous structure modelling based on the crystal structure of wheat (Triticum aestivum) TaCypA-1 (PDB code: $4 \mathrm{E} 1 \mathrm{Q})^{34}$, a cyclophilin sharing $87 \%$ identity with LRT2, revealed that $\mathrm{Gly}^{72}$ is located at the surface of LRT2 immediately adjacent to a $\beta$-sheet (Supplementary Fig. 6b). LRT2 G72A/cyp2-2 recombinant protein, which was less stable than wild-type LRT2 recombinant protein (Supplementary Fig. 7a), showed markedly decreased PPIase activity in catalysing the cis/trans isomerization of the OsIAA11 peptide (Fig. 2b,c; Supplementary Fig 6c,d). LRT2 ${ }^{2} 72 \mathrm{~A} /$ cyp2-2 mutant protein was undetectable in the cyp2-2 mutant $^{17}$ (Supplementary Fig. 7b). Similarly, when transiently expressed in tobacco leaves, no LRT2 ${ }^{\mathrm{G} 72 \mathrm{~A}} / \mathrm{cyp} 2-2$ mutant protein was detected (Supplementary Fig. 7c). These results indicate that $\mathrm{Gly}^{72}$ of LRT2 is important for both its stability and the enzymatic activity.

For the two tandem proline residues specific to the conformation of the Trp ${ }^{104}-$ Pro $^{105}$ and Pro ${ }^{105}$-Pro ${ }^{106}$ motifs, four conformers in the solution were predicted ${ }^{35}$, namely $\mathrm{T}^{105} \mathrm{~T}^{106}$, $\mathrm{T}^{105} \mathrm{C}^{106}, \mathrm{C}^{105} \mathrm{~T}^{106}$ and $\mathrm{C}^{105} \mathrm{C}^{106}$, where $\mathrm{T}$ and $\mathrm{C}$ represented trans and cis conformation, respectively. In the OsIAA11 peptide, the signals of the minor conformers are nearly invisible with serious signal overlapping. Although we observed some exchange 

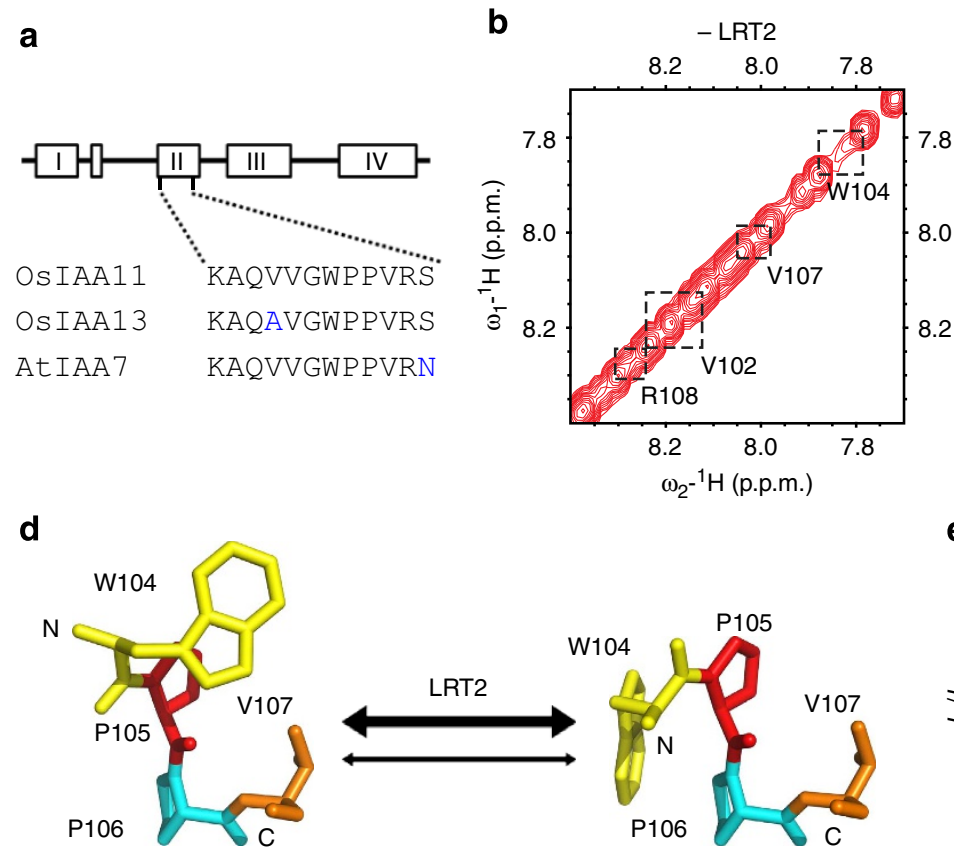

$\mathrm{T}^{105} \mathrm{~T}^{106}$ conformation

$(68 \%)^{*}$

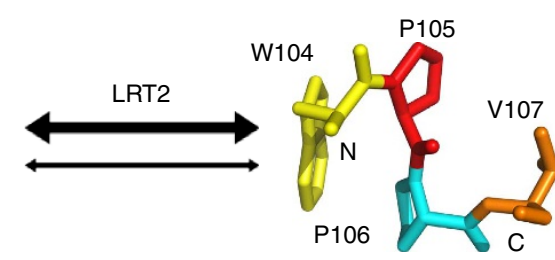

$\mathrm{C}^{105} \mathrm{~T}^{106}$ conformation

$(32 \%)^{\star}$

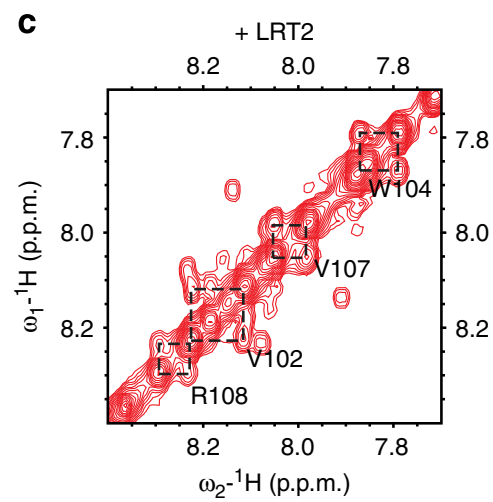

e

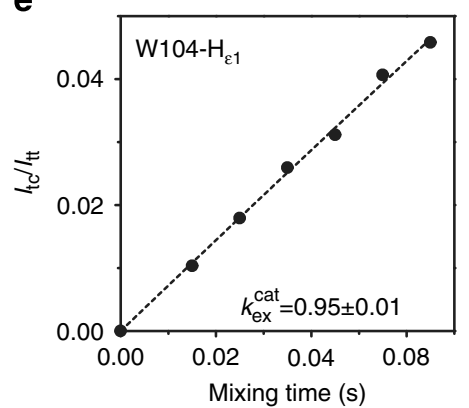

Figure 2 | LTR2 catalyses isomerization of OsIAA11 peptide. (a) Top: the structural features of Aux/IAA proteins. Domains I through IV are highlighted as boxes. Bottom: the alignment of partial sequences in domain II of rice and Arabidopsis Aux/IAAs. Amino-acid residues in OsIAA13 and AtIAA7 that differed with those of OsIAA11 are highlighted in blue. (b,c) Selected region of the $110 \mathrm{~ms}$ mixing time ROESY spectra of the OsIAA11 peptide in the absence (b) and the presence (c) of LRT2 recombinant protein. Signals from the major conformers of residues V102, W104, V107 and R108 are labelled at the positions where exchange cross peaks are expected. (d) Approximate two-state exchange process of the OsIAA11 peptdide. The exchange rate is indicated by the thickness of the double-arrow lines. ${ }^{*}$ indicates the mix populations of the $T^{105} T^{106}+T^{105} C^{106}$ and $C^{105} T^{106}+C^{105} C^{106}$ obtained from the integration of the $\mathrm{H}_{\varepsilon 1}$ signals of $\mathrm{W} 104$ in the $1 \mathrm{D}{ }^{1} \mathrm{H}$ spectrum, respectively, with $\mathrm{T}^{105} \mathrm{~T}^{106}$ and $\mathrm{C}^{105} \mathrm{~T}^{106}$ as major conformers. (e) Dependence of cross/diagonal peak intensity ratios on ROE mixing time in the presence of LRT2 for $\mathrm{H}_{\varepsilon 1}$ of residue W104 of the OsIAA11 peptide. Intensities of diagonal peaks with trans and cis conformations are labelled as $I_{\mathrm{tt}}$ and $I_{\mathrm{cc}}$, respectively. The intensities of exchange peaks resulting from the LRT2-catalysed trans-to-cis and cis-to-trans isomerization are labelled as $I_{\mathrm{tc}}$ and $I_{\mathrm{ct}}$, respectively. Catalysed exchange rate constant $k_{\mathrm{ex}}^{\text {cat }}$ was derived from data points fitted to equation (2) in Methods.

peaks from the minor conformers in the ROESY spectra (Supplementary Fig. 8), it is technically impossible for the signal assignment and difficult to calculate the rate constants of the minor conformers. Considering the lowest cis population of Xaa-Pro bond, where Xaa as a proline ${ }^{35}$ and the type II helix with trans conformation dominating the poly-Pro sequence, the two major conformers of OsIAA11 peptide were deduced as $\mathrm{T}^{105} \mathrm{~T}^{106}$ and $\mathrm{C}^{105} \mathrm{~T}^{106}$, while the minor conformers were predicted as $\mathrm{T}^{105} \mathrm{C}^{106}$ and $\mathrm{C}^{105} \mathrm{C}^{106}$ (Fig. 2d). Since most of Pro ${ }^{105}$-Pro ${ }^{106}$ amide bonds adopt trans conformation, the complex four-state exchange equilibrium could be well approximated by a two-state equilibrium and the exchange rate constant could then be estimated by curve fitting (Fig. 2e). In the presence of LRT2, the peptidyl-prolyl cis/trans conformational exchange was significantly accelerated with $k_{\mathrm{ex}}^{\mathrm{cat}}=0.95 \pm 0.01 \mathrm{~s}^{-1}$ (Fig. 2e), substantially faster than the uncatalysed isomerization rate, which is typically $<0.01 \mathrm{~s}^{-1}$ (refs 36,37). Taken together, these results demonstrate that LRT2 specifically catalyses the cis/trans isomerization of the OsIAA11 peptide.

LRT2 negatively regulates the stability of OsIAA11. Consistent with the above results, the Arabidopsis IAA7 peptide, which shows nearly identical sequence as the OsIAA11 peptide (Fig. 2a), presents as the $\mathrm{C}^{87} \mathrm{~T}^{88}$ (equivalent to $\mathrm{C}^{105} \mathrm{~T}^{106}$ of OsIAA11) conformer in the crystal structure of the TIR1-IAA7 co-receptor ${ }^{6}$. Moreover, the Arabidopsis domain II mutant proteins iaa3/shy2, iaa7/axr2-1 and iaa17/axr3-1, which carry mutations in the highly conserved proline residues (see Supplementary Fig. 2), are incapable of binding the TIR1 receptor $4,5,7,13,15$. These observations, together with the results presented in Figs 1 and 2, raise the possibility that LRT2-catalysed cis/trans isomerization of OsAux/IAAs is critical for the formation of a functional OsTIR1-OsIAA co-receptor. To test this hypothesis, we examined the possible regulatory role of LRT2 on the OsTIR1OsIAA11 interaction by a Co-IP experiment. OsTIR1-Myc protein transiently expressed in tobacco leaves and purified by immunoprecipitation was incubated with His-OsIAA11 recombinant protein and extracts prepared from wild-type or lrt2 plants, and the OsTIR1-OsIAA11 interaction was examined by a Co-IP experiment. In the absence of auxin, OsTIR1-Myc interacted with His-OsIAA11 in a similar affinity when incubated with extracts prepared from wild-type or lrt2 plants (Fig. 3a), a result similar to that previously observed in Arabidopsis ${ }^{15,38}$. This result may be attributed to the presence of a significant portion of the $\mathrm{C}^{105} \mathrm{~T}^{106}$ conformer in the absence of LRT2 (see Fig. 2). The interaction between OsTIR1-Myc and His-OsIAA11 or HisOsIAA13 was substantially enhanced by auxin when incubated with extracts prepared from wild-type plants. However, when incubated with extracts prepared from lrt 2 plants, the interaction between OsTIR1-Myc and His-OsIAA11 or His-OsIAA13 was not responsive to auxin (Fig. 3a), indicating that the LRT2 activity is required for the auxin-dependent OsTIR1-OsIAA interaction. Notably, neither the LRT2 messenger RNA (mRNA) level nor the accumulation of LRT2 protein was regulated by auxin (Supplementary Fig. 9). However, LRT2 protein, which was in a 

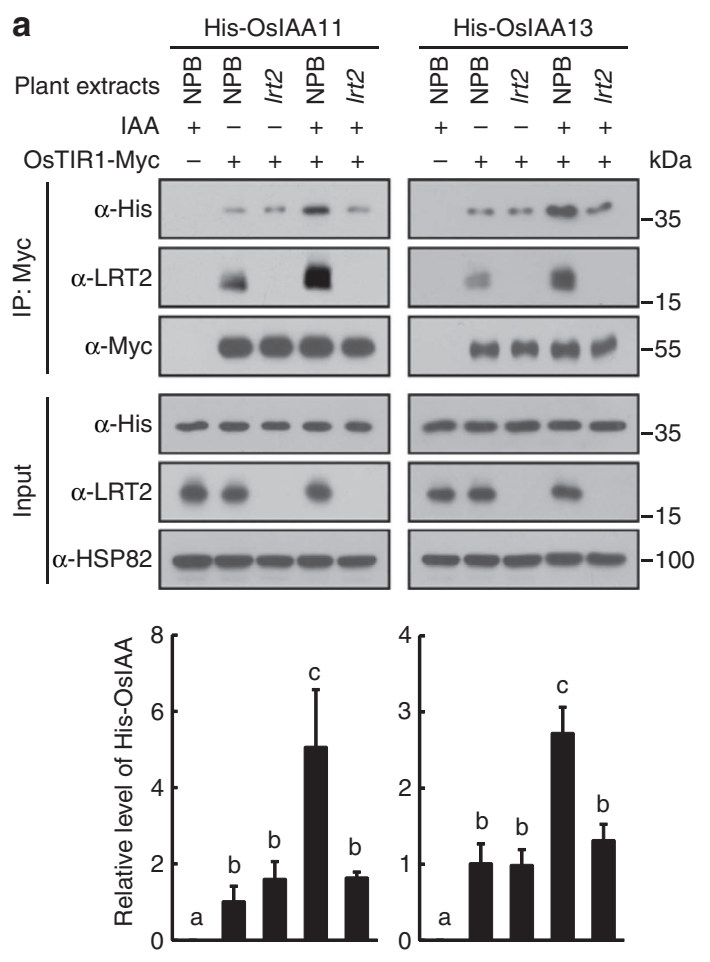

b
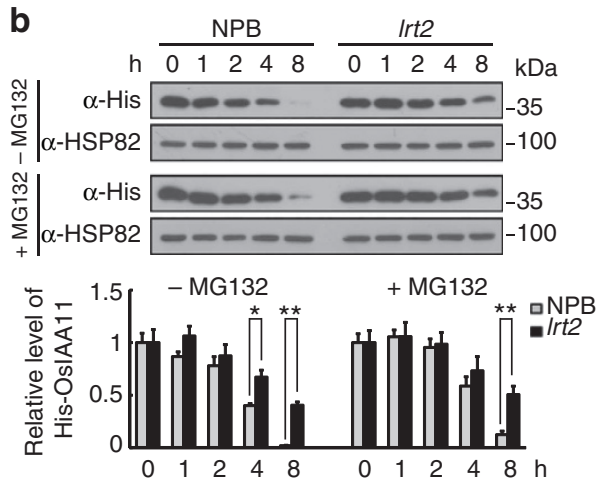

c

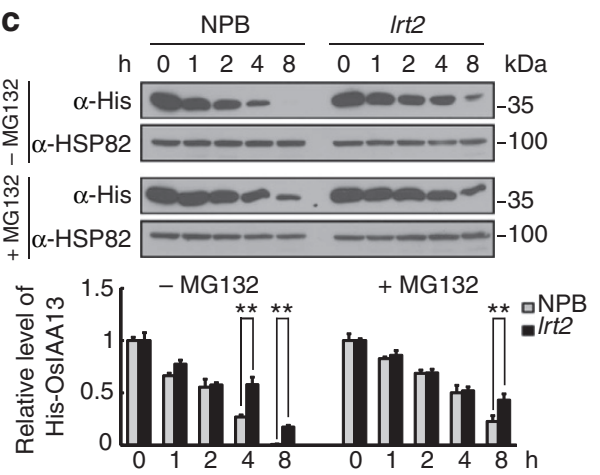

d
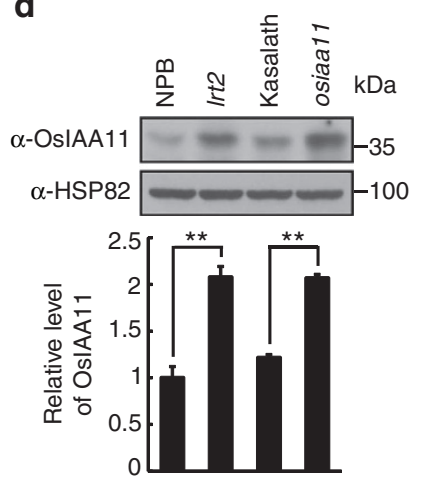

e
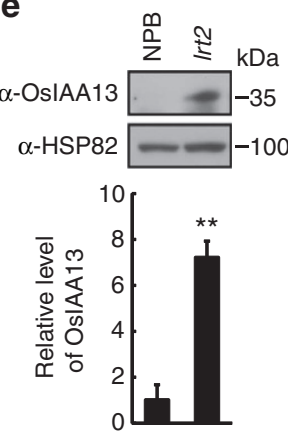

Figure 3 | LRT2 positively regulates the degradation of OsIAAs. (a) LRT2 positively regulates the auxin-induced OsTIR1-OsIAA interaction. OsTIR1-Myc and His-OsIAA11 or His-OsIAA13 proteins were incubated with total protein extracts prepared from the wild-type (NPB) or Irt2 seedlings in the absence or the presence of $50 \mu \mathrm{M} I A A$, and the reaction was immunoprecipitated with an anti-Myc antibody. The immunoprecipitates were then analysed by immunoblotting using antibodies as indicated. Quantitative analysis of the relative level of His-OsIAA11 and His-OsIAA13 is presented below the blots (the average values obtained from three independent experiments). Error bars indicate s.d.; different letters indicate $P<0.05$ (LSD multiple range tests). $(\mathbf{b}, \mathbf{c})$ LRT2 is required for the proteasomal degradation of His-OsIAA11 (b) and His-OsIAA13 (c). His-OsIAA11 or His-OsIAA13 recombinant proteins were incubated with total protein extracts prepared from the wild-type (NPB) or Irt2 seedlings in the absence or the presence of $50 \mu \mathrm{M}$ MG132. Quantitative analysis of the relative level of His-OsIAAs is presented below the blots (the average values obtained from three independent experiments).

(d,e) Immunoblotting analysis of the accumulation of OsIAA11 (d) and OsIAA13 (e) in the wild-type (Nipponbare or NPB and Kasalath), Irt2, and osiaa11 (in the Kasalath background) plants. Quantitative analysis of the relative level of OsIAAs is presented below the blots (the average values obtained from three independent experiments). Equal loading is verified by detecting the HSP82 level in all immunoblotting experiments. Error bars indicate s.d.;

${ }^{\star} P<0.05 ;{ }^{\star \star} P<0.01$, respectively (Student's $t$-test).

same protein complex with OsTIR1 and OsIAA11, was enriched in the complex when treated with auxin (Fig. 3a), implying that the binding of LRT2 to the OsTIR1-OsIAA11 co-receptor is positively regulated by auxin.

To explore the functional significance of the OsTIR1-OsIAALRT2 complex, we next performed an in vitro degradation assay to examine the stability of OsIAA11 and OsIAA13. When incubated with the protein extracts prepared from wild-type or lrt 2 plants, both His-OsIAA11 and His-OsIAA13 recombinant proteins showed the reduced degradation in the absence of LRT2 and the reduced response to MG132, a specific inhibitor of the proteasomal degradation pathway (Fig. 3b,c). Moreover, the accumulation of OsIAA11 and OsIAA13 proteins was increased in $l r t 2$ plants compared with wild type (Fig. $3 \mathrm{~d}$,e). These results indicate that the LRT2-catalysed cis/trans isomerization of OsAux/IAA proteins is critical for the formation of the OsTIR1-OsIAA co-receptor, thereby for the proteasomal degradation of the transcriptional repressors.

OsIAA11 genetically acts downstream of LRT2. Data presented above indicate that LRT2 negatively regulates the accumulation of the auxin signalling repressor OsAux/IAAs. We then reasoned that the reduced expression of the OsAux/IAA genes might partially relieve the lrt2 mutant phenotype. To this end, we generated transgenic rice plants knocking down the expression of OsIAA11 by RNA interference (RNAi). The steady level of OsIAA11 mRNA in the transgenic plants was decreased $\sim 80 \%$ compared with that of wild type (Fig. 4a). Other examined $O s A u x / I A A$ genes were expressed at various levels. Compared with wild-type plants, the expression of OsIAA22 and OsIAA31 was reduced $\sim 50 \%$, whereas the expression of OsIAA14, OsIAA16, OsIAA20 and OsIAA30 was also decreased in the transgenic lines (Supplementary Fig. 10). The target region of OsIAA11 (218bp) showed the highest homology with OsIAA1 (95.1\%), OsIAA30 (92.3\%), OsIAA14 (92.1\%), OsIAA31 (90.9\%) and OsIAA15 $(87.0 \%)$, which did not correlate with the expression levels of these genes in the RNAi-transgenic lines. While the cause of the reduced expression of other non-target OsIAA genes in the RNAi-transgenic plants remains unknown, the expression of OsIAA11 was downregulated to a significantly greater degree compared with wild-type plants (Fig. 4a; Supplementary Fig. 10). Consistently, the accumulation of OsIAA11 protein was also reduced in the RNAitransgenic lines in the lrt 2 mutant background (Fig. $4 \mathrm{~b}$ ). Note that the reduction of the OsIAA11 protein level was less marked than that of OsIAA11 mRNA level in the RNAi-transgenic plants (Fig. 4a,b), which might result from the increased stability of OsIAA 11 protein in the lrt2 mutant background.

The lrt2 mutation caused the markedly reduced number of lateral roots compared with that of wild type $e^{16,17}$ (Fig. 4c,d). 


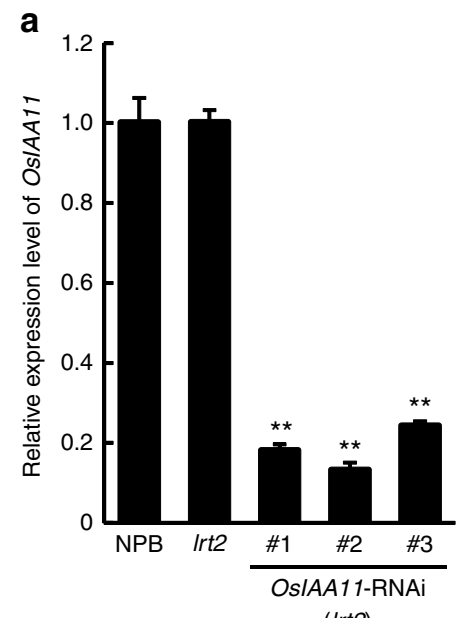

b
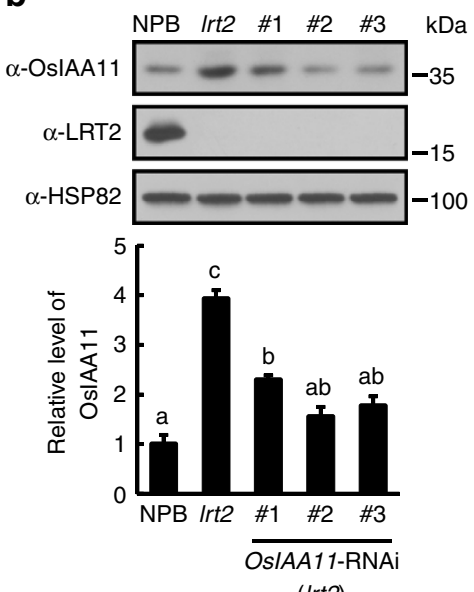

(Irt2)
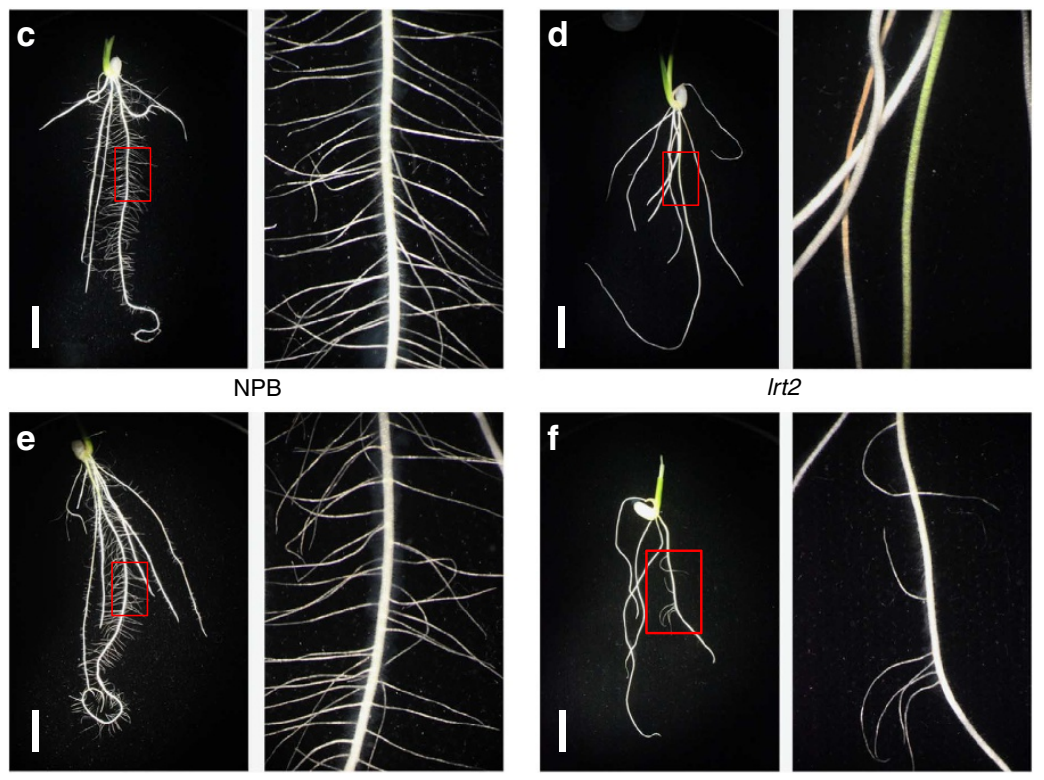

OsIAA11-RNAi (NPB), \#2

OsIAA11-RNAi (Irt2), \#2

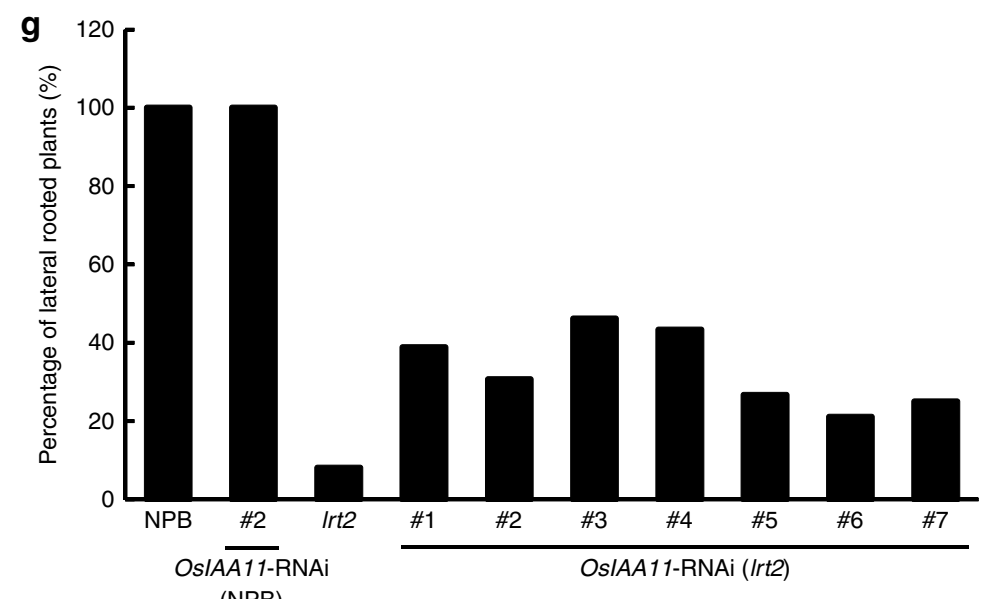

Figure 4 | Knocking down the OsIAA11 expression partially rescues the Irt2 mutant phenotype. (a) Analysis of the expression level of OsIAA11 in RNAitransgenic seedlings (line numbers are given below the graph) by qRT-PCR. Error bars indicate s.d.; ${ }^{\star \star} P<0.01$ (Student's $t$-test). (b) Immunoblotting analysis of the accumulation of OsIAA11 protein in RNAi-transgenic seedlings (line numbers are given below the graph). Equal loading is verified by the analysis of HSP82 protein. Quantitative analysis of the relative level of OsIAA11 protein is presented below the blots. Error bars indicate s.d.; different letters indicate $P<0.05$ (LSD multiple range tests). (c-f) The root phenotype of 10-day-old seedlings with the indicated genotypes. In each panel, the right side shows the enlarged view of the boxed region at the left side. Scale bars, $1 \mathrm{~cm}$. (g) Quantitative analysis of the percentage of lateral rooted plants in 10-dayold seedlings with the indicated genotypes ( $n \geq 30$ for each genotype). Note that, knocking down the OsIAA11 expression in the wild-type background has no detectable effect on lateral root development. 
The knockdown of the OsIAA11 expression in lrt2 partially rescued defective development of lateral roots and the primary roots (Fig. 4e,f; Supplementary Fig. 11). In the RNAi-transgenic plants, the frequency of producing lateral roots was substantially higher than that of $l r t 2$ (Fig. $4 \mathrm{~g}$ ). These results demonstrate that LRT2 genetically acts upstream of OsIAA11 to positively regulate lateral root development.

\section{Discussion}

It has long been known that auxin signalling is activated via a derepression mechanism, in which auxin induces the proteasomal degradation of the signalling repressor Aux/IAAs ${ }^{9,10}$. Yet, it remains uncertain if post-translational modifications are involved in the regulation of the stability of Aux/IAAs ${ }^{15,38}$. We have presented genetic and biochemical evidence demonstrating that the rice cyclophilin LRT2/CYP2 catalyses the peptidyl-prolyl cis/trans isomerization of the OsAux/IAA transcriptional repressors, leading to their binding to OsTIR1 for the targeted proteasomal degradation and eventually activating auxin signalling (Fig. 5).

Considering that the key components, including LRT2-like proteins and Aux/IAAs, are highly conserved in both lower and higher plants and that the loss-of-function mutations in tomato $D G T$ and moss PpDGT cause a similar auxin-insensitive phenotype $^{29,30}$, the cyclophilin-catalysed isomerization of the Aux/IAA transcriptional repressors may represent a general mechanism in regulating auxin signalling in the plant kingdom. In agreement with this notion, LRT2/OsCYP2 was found to directly interact with OsSGT1 (suppressor of G2 allele of skp1) ${ }^{17}$,
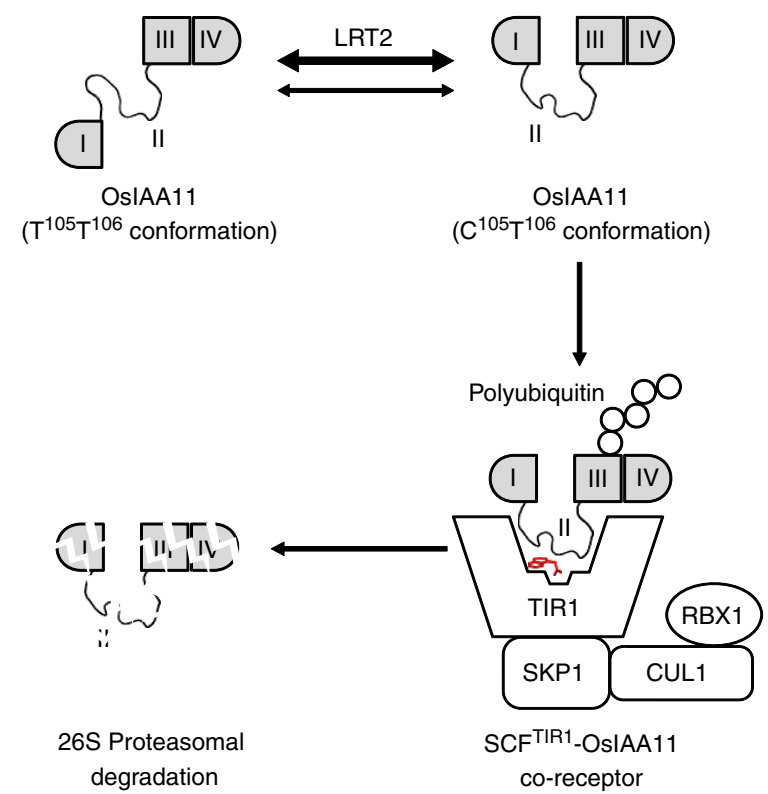

Figure 5 | A proposed model for the LRT2-regulated auxin signalling. The OsIAA11 transcriptional repressor is present as two exchangeable major conformers $\mathrm{T}^{105} \mathrm{~T}^{106}$ and $\mathrm{C}^{105} \mathrm{~T}^{106}$ (indicated by a thin double-arrowed line). The cyclophilin LRT2 catalyses the peptidyl-prolyl cis/trans isomerization at Trp ${ }^{104}$-Pro ${ }^{105}$ peptide bond of OsIAA11, accelerating the formation of the Pro ${ }^{105}$-cis conformer (indicated by a thick double-arrowed line). In the presence of auxin (red molecule), $\mathrm{C}^{105} \mathrm{~T}^{106}$ conformers interact with the SCF $\mathrm{SCR}^{\mathrm{TI} 1}$ complex with a higher affinity than $\mathrm{T}^{105} \mathrm{~T}^{106}$ conformers. The formation of the SCF ${ }^{T I R 1}$-OsIAA11 co-receptor facilitates the ubiquitination and subsequent proteasomal degradation of OsIAA11, which consequently leads to the two-state equilibrium shifting in favour of the formation of $\mathrm{C}^{105} \mathrm{~T}^{106}$ conformers. Domains I through IV are indicated in the OsIAA11 molecule. whose Arabidopsis homologous gene AtSGT1b was identified from a genetic screen for the enhancers of tirl-1 and was characterized as co-chaperone to be required for the SCF ${ }^{\mathrm{TIR} 1}$. mediated degradation of Aux/IAA proteins ${ }^{39}$. Moreover, mutations in the Arabidopsis SIR1 gene, which encodes a protein sharing homology with peptidyl-prolyl isomerase, cause an auxin hypersensitive phenotype, thus providing an additional line of genetic evidence supporting a regulatory role of conformational changes on Aux/IAA proteins ${ }^{40}$. Evidence presented in this study demonstrates that LRT2 modulates the cis/trans isomerization exchanges of OsIAA11 and, possibly, OsIAA13. Two lines of evidence suggest that LRT2 may also target other OsAux/IAA proteins. First, LRT2 interacts with all the tested OsAux/IAA proteins in the luciferase complementation assay, suggesting that these OsAux/IAA proteins are potential targets of LRT2. Second, the knockdown of the OsIAA11 expression only partially rescues the lrt2 mutant phenotype, implying that other downstream factors are likely involved in the LRT2-mediated auxin signalling. In this regard, LRT2 protein may regulate a larger repertoire of OsAux/IAA transcriptional repressors.

CYP have been found to be involved in the signalling of several phytohormones, including auxin, brassinosteroid, gibberellic acid (GA) and jasmonic acid ${ }^{16,17,23-25,41-43}$. Among these and other phytohormones, the activation of the signalling pathways of GA, jasmonic acid and strigolactone also utilize a derepression mechanism by the proteasomal degradation of specific repressors in a manner similar to that of auxin ${ }^{44-48}$. Interestingly, a GA-insensitive dwarf (gaid) mutant of wheat was characterized to be insensitive to exogenous GA, correlated to the elevated levels of the GA signalling repressor Rht/DELLA protein and a putative cyclophilin TaCYP20-2 protein. Moreover, the overexpression of TaCYP20-2 causes the accumulation of excessive amount of Rht/DELLA repressor protein and a dwarf phenotype similar to that of the gaid mutant, suggesting that the putative cyclophilin TaCYP20-2 is involved in DELLA protein degradation during GA signalling ${ }^{42}$. Therefore, the cyclophilinmediated peptidyl-prolyl cis/trans isomerization is not only required for binding of the OsAux/IAAs repressors to OsTIR1 but may also play an important role in regulating other phytohormone signalling pathways.

In summary, we have defined a regulatory role of the rice cyclophilin LRT2 in auxin signalling by mediating the cis/trans isomerization of the Aux/IAA transcriptional repressors, thereby facilitating their proteasomal degradation. Whereas our discovery has revealed a new layer of the regulatory scheme in auxin signalling, the precise molecular mechanism of the auxinpromoted LRT2 activity remains challenging for future studies.

\footnotetext{
Methods

Plant materials and growth conditions. Wild-type rice of Nipponbare, Taichung 65 (Oryza sativa L. ssp. japonica), and Kasalath (Oryza sativa L. ssp. indica) were used in this study. The lrt 2 mutant is in the Nipponbare background and has been previously described ${ }^{16,49}$. The cyp2-2 and osiaa11 mutants are in the Kasalath background and the osiaa 13 mutant is in the Taichung 65 background $^{17,31,32}$, which were kindly provided by Dr Xiaorong Mo, Dr Ping Wu and Dr Yoshiaki Inukai, respectively.

Rice plants were grown in the fields in Beijing, Hangzhou and Hainan with routine management. To analyse the seedling phenotype under the tissue culture condition, seeds were surface sterilized with $75 \%$ ethanol for $5 \mathrm{~min}$, then sterilized with $30 \%$ bleach for $30 \mathrm{~min}$ and rinsed six times with sterile water. Sterilized seeds were plated on MS medium (1/2 MS salts, $1 \%$ sucrose, $0.8 \%$ agar, $\mathrm{pH} 5.9$ ) and grown under $28^{\circ} \mathrm{C}$ at a photoperiod of 16 -h light/8-h dark in a greenhouse.

Genetic transformation of rice was performed as described ${ }^{50}$. In brief, calli derived from rice embryos were infected with agrobacteria cells carrying a target expression vector for 1-2 min. The infected calli were cultured on N6-AS medium (3.981 g $\mathrm{gl}^{-1} \mathrm{~N} 6$ salts, $2 \mathrm{mgl}^{-1} 2,4-\mathrm{D}, 0.3 \mathrm{gl}^{-1}$ casein hydrolysate, $30 \mathrm{gl}^{-1}$ sucrose, $10 \mathrm{gl}^{-1}$ glucose, $15 \mathrm{mgl}^{-1}$ acetosyringone, $4 \mathrm{gl}^{-1}$ phytagel, $\mathrm{pH}$ 5.2) for 3 days at $25^{\circ} \mathrm{C}$. After washing with sterile water containing $500 \mathrm{mgl}^{-1}$ carbenicillin for
} 
three times, the calli were cultured on selection medium $\left(3.981 \mathrm{gl}^{-1} \mathrm{~N} 6\right.$ salts, $2 \mathrm{mgl}^{-1} 2,4-\mathrm{D}, 0.5 \mathrm{gl}^{-1}$ casein hydrolysate, $0.5 \mathrm{gl}^{-1} \mathrm{~L}$-proline, $30 \mathrm{gl}^{-1}$ sucrose, $4 \mathrm{gl}^{-1}$ phytagel, $200 \mathrm{mgl}^{-1}$ carbenicillin, $200 \mathrm{mgl}^{-1}$ cefotaxime, $50 \mathrm{mgl}^{-1}$ hygromycin $\mathrm{B}, \mathrm{pH}$ 5.8) for 2 weeks and the putative positive calli were transferred onto fresh selection medium for an additional 2 weeks. Hygromycin B-resistant calli were then transferred onto differentiation medium $\left(4.4 \mathrm{gl}^{-1} \mathrm{MS}\right.$ salts, $30 \mathrm{gl}^{-1}$ sucrose, $30 \mathrm{gl}^{-1}$ sorbitol, $2 \mathrm{gl}^{-1}$ casein hydrolysate, $0.02 \mathrm{mgl}^{-1} \mathrm{NAA}$, $2 \mathrm{mgl}^{-1}$ kinetin, $4 \mathrm{gl}^{-1}$ phytagel, $50 \mathrm{mgl}^{-1}$ carbenicillin, $50 \mathrm{mgl}^{-1}$ hygromycin $\mathrm{B}, \mathrm{pH}$ 5.8) and cultured for several weeks to regenerate seedlings or plantlets. The regenerated seedlings were then transferred onto rooting medium $\left(2.2 \mathrm{gl}^{-1} \mathrm{MS}\right.$ salts, $0.5 \mathrm{gl}^{-1} \mathrm{~L}$-proline, $0.5 \mathrm{gl}^{-1}$ casein hydrolysate, $30 \mathrm{gl}^{-1}$ sucrose, $0.5 \mathrm{mgl}^{-1}$ IBA, $4 \mathrm{gl}^{-1}$ phytagel, $\mathrm{pH}$ 5.8) and cultured for several weeks.

Plasmid construction. The coding sequences of OsIAA11 and OsIAA13 were PCR amplified from rice complementary DNA (cDNA) and the stop codons were eliminated during PCR. The PCR fragments were cloned into the BamHI and SalI sites of pGEX4T-1 (Amersham Biosciences) and pET28a (Novagen) to generate pGST-OsIAA and pHis-OsIAA expression vectors, respectively. The pGST-LRT2 and pHis-LRT2 constructs were generated in a similar way using the EcoRI and Sall sites.

To create the pGADT7-OsIAA constructs, the coding sequences of OsIAA11 and OsIAA13 were PCR amplified from rice cDNA and the stop codons were eliminated. The PCR fragments were cloned into the EcoRI and SalI sites of pGADT7 (Clontech). The pGBKT7-LRT2 construct was generated in a similar way.

The coding sequence of $L R T 2$ was PCR amplified from rice cDNA and the stop codons were eliminated. The gene was inserted in the KpnI and SalI sites of pCAMBIA-nLUC ${ }^{51}$. The coding sequences of OsIAA1, OsIAA11, OsIAA13, OsIAA20, OsIAA22, OsIAA23 and OsIAA30 were PCR amplified from rice cDNA and the stop codons were eliminated. The OsIAA20 and OsIAA30 genes were cloned into the KpnI and PstI sites, and the others were cloned into the KpnI and Sall sites of pCAMBIA-CLUC ${ }^{51}$.

The OsIAA11-RNAi (OSIAA11-RNAi) vector was constructed by sequentially inserting two inverted copies of a 218-base pair (bp) cDNA fragment of OsIAA11 (nucleotides 143-361; the first nucleotide of the putative translation codon was referred to as +1 ) into the pTCK303 vector ${ }^{52}$ using the BamHI/KpnI and SpeI/ SacI sites, respectively.

The pWM101 vector ${ }^{53}$ was used to construct 35S::OsIAA11-FLAG, 35S::OsIAA13-FLAG and 35S::LRT2-Myc expression vectors. The coding sequences of OsIAA11, OsIAA13 and LRT2 were PCR amplified from rice cDNA and the stop codons were eliminated. The fragments were cloned into the KpnI and XmaI sites of pBluescript SK (Stratagene), in-frame fused to a FLAG tag or $6 \times$ Myc tag to generate fusion genes, which were cloned into the KpnI/XbaI sites of pWM101 vector.

To construct 35S::OsTIR1-Myc-NOS vector, the 35S promoter fragment, a $6 \times$ Myc tag and the NOS terminator sequences were ligated into the binary vector pCAMBIA1300 using EcoRI/SacI, SalI/PstI and PstI/HindIII sites, respectively. The coding sequence of OsTIR1 (lacking the stop codon) was inserted into the pCAMBIA1300-35S-Myc-NOS vector using the BamHI and SalI sites.

Site-directed mutagenesis was performed using the Easy Mutagenesis System (TransGen Biotech, Beijing) according the manufacturer's instructions.

All the binary expression vectors were introduced into Agrobacterium tumefaciens strain EHA105 and then used for the transformation of rice or the infiltration of tobacco leaves. All primers used for plasmid construction are listed in Supplementary Table 1 . The accession numbers of all genes analyzed in this study are listed in Supplementary Table 2.

Quantitative reverse transcription-PCR. Total RNA was prepared using the RNAprep Pure Plant RNA Purification Kit (Tiangen Biotech). Quantitative reverse transcription-PCR (qRT-PCR) was performed using the UltraSYBR Mixture (CWBIO) according to the manufacturer's instructions. The reactions were run in a CFX96 REAL-Time PCR Detection System (Bio-Rad). The relative expression level of the target genes was analysed with the delta-delta Ct method and normalized to the expression level of OsACTIN2. All of the experiments were repeated for at least twice (two biological repeats with three technical repeats for each experiment). The primers used for qRT-PCR are listed in Supplementary Table 1.

Antibody preparation and immunoblotting. The anti-LRT2 polyclonal antibody has been previously described ${ }^{16}$. To prepare anti-OsIAA11 and anti-OsIAA13 antibodies, the full-length cDNA fragments were used to produce recombinant proteins tagged with $6 \times$ His. The purified $6 \times$ His-tagged recombinant proteins were used to immunize mice and rabbits, respectively. Immunoblotting was performed as described previously ${ }^{54}$. Total cellular proteins were prepared by grinding plant materials in liquid nitrogen and then extracted in grinding buffer ( $50 \mathrm{mM}$ Tris- $\mathrm{HCl}, \mathrm{pH} 8.0,150 \mathrm{mM} \mathrm{NaCl}, 1 \%$ Nonidet P- $40,0.5 \%$ sodium deoxycholate, $0.1 \%$ SDS and $1 \mathrm{mM}$ phenylmethylsulfonyl fluoride). After centrifugation twice at $14,000 \mathrm{~g}$ for $15 \mathrm{~min}$ at $4^{\circ} \mathrm{C}$, the supernatant was collected and then subjected to SDS-polyacrylamide gel electrophoresis. After the run, proteins were electrically transferred onto a polyvinylidene difluoride membrane, and then detected with a primary antibody of indicated (usually at 1:5,000 dilution). The blot was incubated with a secondary antibody (horseradish peroxidase-conjugated goat anti-rabbit IgG or horseradish peroxidase -conjugated goat anti-mouse IgG; Beijing Dingguo Changsheng Biotechnology) at 1:50,000 dilution. The signal was detected using a SuperSignal Western Femto Maximun Sensitivity Substrate kit (Thermo Scientific, Cat no.: 34096) according to the manufacturer's instructions. Rice HSP82 was used as a loading control. In most immunoblotting experiments, the blot was first probed with an antibody specific to a target protein and then stripped, followed by re-probing the blot with anti-HSP 82 antibody. Occasionally, two technical replicates (gels) were simultaneously performed and then analysed with antibodies against the specific targets and HSP82, respectively (Fig. 3d). The target bands and loading control bands were quantified using NIH ImageJ (version 1.42q, http://rsbweb.nih.gov/ij/) and the mean values of 3-5 independent experiments were presented with statistical analysis (LSD multiple range tests for Fig. 3a and Fig. 4b; Student's $t$-test for all other experiments) of significant differences when applicable. Uncropped versions of all blots are presented in Supplementary Figures 12-14.

Yeast two-hybrid assay. The coding sequences of OsIAA11 and OsIAA13 were cloned into the prey vector pGADT7 (Clontech). The LRT2 full-length coding sequence was cloned into the bait vector pGBKT7 (Clontech). The bait and prey constructs were co-transformed into the yeast (Saccharomyces cerevisiae) strain AH109 (Clontech). The transformants were grown on SD-Leu/-Trp plates for 3-5 days at $30^{\circ} \mathrm{C}$. The interactions between bait and prey were examined on the control media-LT (SD-Leu/-Trp) and selective media-LTHA (SD-Leu/-Trp/-His/-Ade) supplemented with $\mathrm{X}$ - $\alpha$-gal by incubating the plates at $30^{\circ} \mathrm{C}$ for $3-5$ days.

$\mathbf{L C I}$ assay. The detection of protein-protein interactions by the LCI assay was performed as described ${ }^{51}$. LRT2 and OsIAA cDNA fragments were in-frame fused with $n L U C$ and $C L U C$ in the pCAMBIA-nLUC and pCAMBIA-cLUC vectors ${ }^{51}$, respectively. The resulting binary expression vectors were transformed into Agrobacterium strain EHA105. Agrobacterial cells carrying various expression vectors were co-infiltrated into Nicotiana benthamiana (N. benthamiana) leaves with appropriate controls. After the infiltration, plants were placed at $22^{\circ} \mathrm{C}$ for $72 \mathrm{~h}$ and the fluorescent images were collected with a low-light cooled charge-coupled device (CCD) imaging apparatus (Andor iXon). The experiment was repeated three times with independent biological replicates.

Pull-down assay. Protein pull-down assay was performed as described ${ }^{55}$ with minor modifications. In brief, purified GST, GST-LRT2, His-OsIAA11 and HisOsIAA13 proteins were immobilized on GST beads (Glutathione Sepharose 4B; GE Healthcare). Immobilized Sepharose beads containing $2 \mu \mathrm{g}$ GST or GST-LRT2 fusion proteins were mixed with $2 \mu \mathrm{g}$ His-tagged proteins and then incubated at $4^{\circ} \mathrm{C}$ for $2 \mathrm{~h}$. The beads were collected by centrifugation and then washed six times with washing buffer (10 $\mathrm{mM}$ phosphate buffer saline, $\mathrm{pH} 7.4,150 \mathrm{mM} \mathrm{NaCl}, 0.2 \%$ Triton X-100 and $1 \mathrm{mM}$ phenylmethanesulfonyl fluoride) at $4{ }^{\circ} \mathrm{C}$. The beads were resuspended in SDS-polyacrylamide gel electrophoresis sample buffer and then analysed by immunoblotting.

In vitro turnover assay. The analysis of OsIAA protein degradation in vitro was performed as described ${ }^{56}$ with minor modifications. In brief, total protein extracts were prepared from 9-day-old rice seedlings grown in MS/sucrose medium using ice-cold extraction buffer ( $50 \mathrm{mM}$ Tris-HCl, $\mathrm{pH} 7.5,150 \mathrm{mM} \mathrm{NaCl}, 0.01 \%$ Triton $\mathrm{X}-100$ and $1 \mathrm{mM}$ phenylmethanesulfonyl fluoride). The resulting homogenates were centrifuged at $14,000 \mathrm{~g}$ for $15 \mathrm{~min}$ at $4{ }^{\circ} \mathrm{C}$. The crude extracts ( $1 \mathrm{mg}$ proteins) were mixed with $2 \mu \mathrm{g}$ of purified His-OsIAA11 or His-OsIAA13 recombinant proteins in a total volume of $1 \mathrm{ml}$ containing $50 \mu \mathrm{M}$ MG132 or an equal volume DMSO as a control. The mixture was incubated at $4{ }^{\circ} \mathrm{C}$ with gentle agitation and $20 \mu \mathrm{l}$ of each sample was collected at the indicated time points and then analysed by immunoblotting.

Co-immunoprecipitation experiments. The Co-IP experiments were performed according to the previously described methods with minor modifications ${ }^{57}$. To prepare total cellular proteins, plant samples were grinded in liquid nitrogen, and then extracted in grinding buffer $(50 \mathrm{mM}$ Tris- $\mathrm{HCl}, \mathrm{pH} 7.5,150 \mathrm{mM} \mathrm{NaCl}, 10 \mathrm{mM}$ $\mathrm{MgCl}_{2}, 10 \%$ glycerol, $0.1 \%$ Nonidet $\mathrm{P}-40,1 \mathrm{mM}$ phenylmethylsulfonyl fluoride and $10 \mu \mathrm{M}$ MG132). The extracts were cleared by centrifugation at $14,000 \mathrm{~g}$ for $15 \mathrm{~min}$ at $4^{\circ} \mathrm{C}$.

For the Co-IP experiments using rice protein samples, the extracts containing $1-2 \mathrm{mg}$ of proteins were incubated with $10 \mu \mathrm{l}$ of the anti-OsIAA11 or antiOsIAA13 antibodies for $1 \mathrm{~h}$ at $4{ }^{\circ} \mathrm{C}$ with gentle shaking. Protein A-agarose beads $\left(100 \mu \mathrm{l}\right.$; Sigma-Aldrich) were added and incubated for an additional $2-3 \mathrm{~h}$ at $4{ }^{\circ} \mathrm{C}$. The immunoprecipitates were washed six times with $1 \mathrm{ml}$ washing buffer (grinding buffer without MG132) and then used for immunoblotting.

For the Co-IP experiments using samples prepared from Agrobacteriuminfiltrated $N$. benthamiana leaves, the extracts containing $1-2 \mathrm{mg}$ of total proteins were incubated with $10 \mu \mathrm{l}$ of protein A-agarose (Sigma-Aldrich) for $1 \mathrm{~h}$ at $4^{\circ} \mathrm{C}$, followed by adding $100 \mu \mathrm{l}$ of anti-DDDDK-tag pAb-Agarose beads (MBL 
International) and incubating for an additional $2-3 \mathrm{~h}$ at $4^{\circ} \mathrm{C}$. The pellets were washed six times with $1 \mathrm{ml}$ washing buffer and then used for immunoblotting.

To analyse the OsTIR1 receptor complex ${ }^{15,38}$, the OsTIR1-Myc fusion protein was transiently expressed in tobacco leaves by the Agrobacterium-mediated transformation and purified by immunoprecipitation using an anti-cMyc antibody (EarthOx Life Science). In brief, the crude protein extracts were prepared using a slightly modified extraction buffer $(50 \mathrm{mM}$ Tris- $\mathrm{HCl}, \mathrm{pH} 7.5,150 \mathrm{mM} \mathrm{NaCl}$, $10 \mathrm{mM} \mathrm{MgCl}, 10 \%$ glycerol, $0.1 \%$ Nonidet P-40, 1 mM PMSF and $10 \mu \mathrm{M}$ MG132) from tobacco leaves infiltrated with agrobacteria cells containing a 35S:OsTIR1-Myc expression vector, and the extracts were cleared by centrifugation at $14,000 \mathrm{~g}$ for $15 \mathrm{~min}$. The protein extracts (1-2 $\mathrm{mg}$ of total proteins) were incubated with $5 \mu \mathrm{l}$ of anti-cMyc antibodies for $1 \mathrm{~h}$ at $4{ }^{\circ} \mathrm{C}$, and then $100 \mu \mathrm{l}$ of protein A-agarose (SigmaAldrich) was added and incubated for an additional $2-3 \mathrm{~h}$ at $4{ }^{\circ} \mathrm{C}$. After washing six times, the beads were mixed with protein extracts prepared from wild-type (Nipponbare) or $l r t 2$ seedlings (1-2 mg of proteins), $2 \mu \mathrm{g}$ of purified His-OsIAA11 or His-OsIAA13 recombinant proteins and IAA at the indicated concentrations. The reaction was run at $4{ }^{\circ} \mathrm{C}$ for $2-3 \mathrm{~h}$ with gentle shaking, and the beads were washed six times and subsequently used for immunoblotting.

NMR analysis. The NMR analysis was performed according to the previously described methods with minor modifications ${ }^{26}$. All NMR experiments were performed on an Agilent $600 \mathrm{MHz}$ NMR spectrometer equipped with a cold probe at $25^{\circ} \mathrm{C}$. The lyophilized peptide powder (GL Biochem) was dissolved in $20 \mathrm{mM}$ phosphate buffer ( $\mathrm{pH}$ 6.5) with or without $0.05 \mathrm{mM}$ LRT2 recombinant proteins, and the final concentration of the peptide was $2 \mathrm{mM}$. For all the ROESY experiments, $1,024 \times 256$ complex points were collected with the spectra widths of $7668.7 \mathrm{~Hz}$ in both dimensions. For each sample of LRT2 protein, a series of ROESY experiments were run with mixing time of 30, 50, 70, 90, 110 and $130 \mathrm{~ms}$. For the control samples (samples without LRT2 protein), the longest mixing time in the series was used to carry out the ROESY experiment for comparison. All ROESY spectra were collected using 16 scans per fid with spinlock field strength of $2.8 \mathrm{KHz}$ and $1 \mathrm{~s}$ delay time. For the assigning of the signals, TOCSY spectra were collected using $80 \mathrm{~ms}$ mixing time and 8 scans per fid with a spinlock field strength of $7 \mathrm{KHz}$. Signals of the trans and cis conformers were distinguished using the NOEs between the proline residue and its preceding residue ${ }^{58}$. The ratios of cross to diagonal peak intensities for cis and trans conformations in the ROESY spectra were dependent on the total exchange rate constant $k_{\text {ex }}$ of a two-state exchange process (cis $\leftrightarrow$ trans) and the mixing time of the ROESY experiments $\left(t_{\mathrm{m}}\right)$. The exchange rate constants were obtained by fitting one of the following equations using different ratios with different mixing times ${ }^{59,60}$ :

$$
\begin{aligned}
& I_{c t} / I_{c c}=a \cdot k_{e x} \cdot\left[\exp \left(k_{e x} t_{m}\right)-1\right] /\left[b \cdot k_{e x} \cdot \exp \left(k_{e x} t_{m}\right)+a \cdot k_{e x}\right] \\
& I_{t c} / I_{t t}=b \cdot k_{e x} \cdot\left[\exp \left(k_{\text {ex }} t_{\mathrm{m}}\right)-1\right] /\left[a \cdot k_{\text {ex }} \cdot \exp \left(k_{\text {ex }} t_{\mathrm{m}}\right)+b \cdot k_{\text {ex }}\right]
\end{aligned}
$$

where $I_{\mathrm{cc}}$ and $I_{\mathrm{tt}}$ are the diagonal peak intensities for the cis and trans conformers, and $I_{\mathrm{ct}}$ and $I_{\mathrm{tc}}$ are the cross peak intensities for the cis-to-trans and trans-to-cis exchange. $a$ and $b$ were determined from the normalized integration of separated peaks from cis and trans conformer of a $1 \mathrm{D}^{1} \mathrm{H}$ spectrum and the catalysed forward $\left(k_{\mathrm{ct}}^{\mathrm{cat}}\right)$ and backward $\left(k_{\mathrm{tc}}^{\mathrm{cat}}\right)$ rates equal to $a^{*} k_{\mathrm{ex}}$ and $b^{*} k_{\mathrm{ex}}$ respectively. The total exchange rate constant $k_{\mathrm{ex}}=k_{\mathrm{ct}}^{\mathrm{cat}}+k_{\mathrm{tc}}^{\mathrm{cat}}$.

Structure modelling. Modelling of the 3D structure of LRT2 was performed as previously described ${ }^{54}$ through the SWISS-Model server (http://swissmodel.expasy.org) using the crystal structure of wheat TaCypA-1 (PDB ID: 4E1Q) as a template ${ }^{34}$. Molecular graphics were presented using PyMOL software (http://www.pymol.org/).

\section{References}

1. Dharmasiri, N. \& Estelle, M. Auxin signaling and regulated protein degradation. Trends Plant Sci. 9, 302-308 (2004).

2. Peer, W. A. From perception to attenuation: auxin signalling and responses. Curr. Opin. Plant Biol. 16, 561-568 (2013).

3. Wang, R. \& Estelle, M. Diversity and specificity: auxin perception and signaling through the TIR1/AFB pathway. Curr. Opin. Plant Biol. 21, 51-58 (2014).

4. Kepinski, S. \& Leyser, O. The Arabidopsis F-box protein TIR1 is an auxin receptor. Nature 435, 446-451 (2005).

5. Dharmasiri, N., Dharmasiri, S. \& Estelle, M. The F-box protein TIR1 is an auxin receptor. Nature 435, 441-445 (2005).

6. Tan, X. et al. Mechanism of auxin perception by the TIR1 ubiquitin ligase. Nature 446, 640-645 (2007).

7. Gray, W. M., Kepinski, S., Rouse, D., Leyser, O. \& Estelle, M. Auxin regulates SCF $^{T I R 1}$-dependent degradation of AUX/IAA proteins. Nature 414, 271-276 (2001).

8. Tiwari, S. B., Wang, X.-J., Hagen, G. \& Guilfoyle, T. J. AUX/IAA proteins are active repressors, and their stability and activity are modulated by auxin. Plant Cell 13, 2809-2822 (2001)

9. Liscum, E. \& Reed, J. W. Genetics of Aux/IAA and ARF action in plant growth and development. Plant Mol. Biol. 49, 387-400 (2002).

10. Reed, J. W. Roles and activities of Aux/IAA proteins in Arabidopsis. Trends Plant Sci. 6, 420-425 (2001)
11. Mockaitis, K. \& Estelle, M. Auxin receptors and plant development: a new signaling paradigm. Annu. Rev. Cell Dev. Biol. 24, 55-80 (2008).

12. Ramos, J. A., Zenser, N., Leyser, O. \& Callis, J. Rapid degradation of auxin/ indoleacetic acid proteins requires conserved amino acids of domain II and is proteasome dependent. Plant Cell 13, 2349-2360 (2001).

13. Tian, Q., Nagpal, P. \& Reed, J. W. Regulation of Arabidopsis SHY2/IAA3 protein turnover. Plant J. 36, 643-651 (2003).

14. Yang, X. et al. The IAA1 protein is encoded by AXR5 and is a substrate of SCF ${ }^{\text {TIR1 }}$. Plant J. 40, 772-782 (2004).

15. Dharmasiri, N., Dharmasiri, S., Jones, A. M. \& Estelle, M. Auxin action in a cell-free system. Curr. Biol. 13, 1418-1422 (2003).

16. Zheng, H. et al. LATERAL ROOTLESS2, a cyclophilin protein, regulates lateral root initiation and auxin signaling pathway in rice. Mol. Plant 6, 1719-1721 (2013).

17. Kang, B. et al. OsCYP2, a chaperone involved in degradation of auxinresponsive proteins, plays crucial roles in rice lateral root initiation. Plant J. 74, 86-97 (2013)

18. Romano, P. G. N., Horton, P. \& Gray, J. E. The Arabidopsis cyclophilin gene family. Plant Physiol. 134, 1268-1282 (2004).

19. Lu, K. P., Finn, G., Lee, T. H. \& Nicholson, L. K. Prolyl cis-trans isomerization as a molecular timer. Nat. Chem. Biol. 3, 619-629 (2007).

20. Gasser, C. S., Gunning, D. A., Budelier, K. A. \& Brown, S. M. Structure and expression of cytosolic cyclophilin/peptidyl-prolyl cis-trans isomerase of higher plants and production of active tomato cyclophilin in Escherichia coli. Proc. Natl Acad. Sci. USA 87, 9519-9523 (1990).

21. Kumari, S., Roy, S., Singh, P., Singla-Pareek, S. \& Pareek, A. Cyclophilins: proteins in search of function. Plant Signal Behav. 8, e22734 (2013).

22. Vasudevan, D. et al. Plant immunophilins: a review of their structure-function relationship. Biochim. Biophys. Acta doi:10.1016/j.bbagen.2014.12.017 (19 December 2014).

23. Trupkin, S. A., Mora-García, S. \& Casal, J. J. The cyclophilin ROC1 links phytochrome and cryptochrome to brassinosteroid sensitivity. Plant J. 71, 712-723 (2012).

24. Ma, X., Song, L., Yang, Y. \& Liu, D. A gain-of-function mutation in the ROC1 gene alters plant architecture in Arabidopsis. New Phytol. 197, 751-762 (2013).

25. Zhang, Y. et al. The cyclophilin CYP20-2 modulates the conformation of BRASSINAZOLE-RESISTANT1, which binds the promoter of FLOWERING LOCUS D to regulate flowering in Arabidopsis. Plant Cell 25, 2504-2521 (2013).

26. Wang, Y., Liu, C., Yang, D., Yu, H. \& Liou, Y.-C. Pin1At encoding a peptidylprolyl cis/trans isomerase regulates flowering time in Arabidopsis. Mol. Cell 37, 112-122 (2010).

27. $\mathrm{Li}, \mathrm{M}$. et al. Proline isomerization of the immune receptor-interacting protein RIN4 by a cyclophilin inhibits effector-triggered immunity in Arabidopsis. Cell Host Microbe. 16, 473-483 (2014).

28. Oh, K., Ivanchenko, M., White, T. J. \& Lomax, T. The diageotropica gene of tomato encodes a cyclophilin: a novel player in auxin signaling. Planta 224, 133-144 (2006).

29. Ivanchenko, M. G., Coffeen, W. C., Lomax, T. L. \& Dubrovsky, J. G. Mutations in the Diageotropica (Dgt) gene uncouple patterned cell division during lateral root initiation from proliferative cell division in the pericycle. Plant J. 46, 436-447 (2006)

30. Lavy, M., Prigge, M. J., Tigyi, K. \& Estelle, M. The cyclophilin DIAGEOTROPICA has a conserved role in auxin signaling. Development 139, 1115-1124 (2012).

31. Zhu, Z.-X. et al. A gain-of-function mutation in OsIAA11 affects lateral root development in rice. Mol. Plant 5, 154-161 (2012).

32. Kitomi, Y., Inahashi, H., Takehisa, H., Sato, Y. \& Inukai, Y. OsIAA13-mediated auxin signaling is involved in lateral root initiation in rice. Plant Sci. 190, 116-122 (2012).

33. Jun, N. et al. OsIAA23-mediated auxin signaling defines postembryonic maintenance of QC in rice. Plant J. 68, 433-442 (2011).

34. Sekhon, S. S. et al. Structural and biochemical characterization of the cytosolic wheat cyclophilin TaCypA-1. Acta Crystallogr. D Biol. Crystallogr. 69, 555-563 (2013).

35. Reimer, U. et al. Side-chain effects on peptidyl-prolyl cis/trans isomerisation. J. Mol. Biol. 279, 449-460 (1998).

36. Schönbrunner, E. R. et al. Catalysis of protein folding by cyclophilins from different species. J. Biol. Chem. 266, 3630-3635 (1991).

37. Endrich, M. M., Gehrig, P. \& Gehring, H. Maturation-induced conformational changes of HIV-1 capsid protein and identification of two high affinity sites for cyclophilins in the C-terminal domain. J. Biol. Chem. 274, 5326-5332 (1999).

38. Kepinski, S. \& Leyser, O. Auxin-induced SCF ${ }^{\mathrm{TIR} 1}$-Aux/IAA interaction involves stable modification of the SCF ${ }^{\mathrm{TIR} 1}$ complex. Proc. Natl Acad. Sci. USA 101, 12381-12386 (2004). 
39. Gray, W. M., Muskett, P. R., Chuang, H.-W. \& Parker, J. E. Arabidopsis SGT1b is required for $\mathrm{SCF}^{\mathrm{TIR} 1}$-mediated auxin response. Plant Cell 15, 1310-1319 (2003).

40. Zhao, Y., Dai, X., Blackwell, H. E., Schreiber, S. L. \& Chory, J. SIR1, an upstream component in auxin signaling identified by chemical genetics. Science 301, 1107-1110 (2003).

41. Pérez-Pérez, J. M., Ponce, M. R. \& Micol, J. L. The ULTRACURVATA2 gene of Arabidopsis encodes an FK506-binding protein involved in auxin and brassinosteroid signaling. Plant Physiol. 134, 101-117 (2004).

42. Li, B. et al. Integrative study on proteomics, molecular physiology, and genetics reveals an accumulation of cyclophilin-like protein, TaCYP20-2, leading to an increase of Rht protein and dwarf in a novel GA-insensitive mutant (gaid) in wheat. J. Proteome Res. 9, 4242-4253 (2010).

43. Park, S.-W. et al. Cyclophilin 20-3 relays a 12-oxo-phytodienoic acid signal during stress responsive regulation of cellular redox homeostasis. Proc. Natl. Acad. Sci. USA 110, 9559-9564 (2013).

44. Hirano, K., Ueguchi-Tanaka, M. \& Matsuoka, M. GID1-mediated gibberellin signaling in plants. Trends Plant Sci. 13, 192-199 (2008).

45. Sun, T.-p. The molecular mechanism and evolution of the GA-GID1-DELLA signaling module in plants. Curr. Biol. 21, R338-R345 (2011).

46. Pérez, A. C. \& Goossens, A. Jasmonate signalling: a copycat of auxin signalling? Plant Cell Environ. 36, 2071-2084 (2013).

47. Jiang, L. et al. DWARF 53 acts as a repressor of strigolactone signalling in rice. Nature 504, 401-405 (2013).

48. Zhou, F. et al. D14-SCF ${ }^{\mathrm{D} 3}$-dependent degradation of D53 regulates strigolactone signalling. Nature 504, 406-410 (2013).

49. Wang, H., Taketa, S., Miyao, A., Hirochika, H. \& Ichii, M. Isolation of a novel lateral-rootless mutant in rice (Oryza sativa L.) with reduced sensitivity to auxin. Plant Sci. 170, 70-77 (2006).

50. Hiei, Y., Ohta, S., Komari, T. \& Kumashiro, T. Efficient transformation of rice (Oryza sativa L.) mediated by Agrobacterium and sequence analysis of the boundaries of the T-DNA. Plant J. 6, 271-282 (1994).

51. Chen, H. et al. Firefly luciferase complementation imaging assay for proteinprotein interactions in plants. Plant Physiol. 146, 368-376 (2008).

52. Wang, Z. et al. A practical vector for efficient knockdown of gene expression in rice (Oryza sativa L.). Plant Mol. Biol. Rep. 22, 409-417 (2004).

53. Ding, Y.-H., Liu, N.-Y., Tang, Z.-S., Liu, J. \& Yang, W.-C. Arabidopsis GLUTAMINE-RICH PROTEIN23 is essential for early embryogenesis and encodes a novel nuclear PPR motif protein that interacts with RNA polymerase II subunit III. Plant Cell 18, 815-830 (2006).

54. Feng, J. et al. S-nitrosylation of phosphotransfer proteins represses cytokinin signaling. Nat. Commun. 4, 1529 (2013).

55. Liu, H. et al. Photoexcited CRY2 interacts with CIB1 to regulate transcription and floral initiation in Arabidopsis. Science 322, 1535-1539 (2008)
56. Nakamura, A. et al. Production and characterization of auxin-insensitive rice by overexpression of a mutagenized rice IAA protein. Plant J. 46, 297-306 (2006).

57. Dharmasiri, N. et al. Plant development is regulated by a family of auxin receptor F box proteins. Dev. Cell 9, 109-119 (2005).

58. Ramelot, T. A. \& Nicholson, L. K. Phosphorylation-induced structural changes in the amyloid precursor protein cytoplasmic tail detected by NMR. J. Mol. Biol. 307, 871-884 (2001).

59. Pastorino, L. et al. The prolyl isomerase Pin1 regulates amyloid precursor protein processing and amyloid- $\beta$ production. Nature 440, 528-534 (2006)

60. Pastorino, L. et al. The prolyl isomerase Pin1 regulates amyloid precursor protein processing and amyloid- $\beta$ production. Nature 446, 342-342 (2007)

\section{Acknowledgements}

We thank Dr Xiaorong Mo for cyp2-2 mutant seeds; Dr Ping Wu for osiaa11 mutant seeds; Dr Yoshiaki Inukai for osiaa13 seeds; Drs Kang Chong, Jian-Min Zhou and Wei-Cai Yang for expression vectors; Dr Kang Chong for sharing the unpublished data Drs Makoto Matsuoka and Yuling Jiao for critical reading the manuscript and valuable comments; Dr Neing Yan and members of Zuo laboratory for helpful discussion. This work was supported by grants from the National Natural Science Foundation of China (91217302), the Ministry of Science and Technology of China (2014CB943402) and State Key Laboratory of Plant Genomics (Y260022512).

\section{Author contributions}

H.J., X.Y., X.L., Q.Q., J.L. and J.Zuo. designed the experiments. H.J. and X.Y. performed the majority of the experiments, assisted by J.Zhang., X.L., H.Z., G.D., J.N. and J.F. All authors analysed the data and commented on the manuscript. H.J., X.Y., X.L., B.X., J.L. and J.Zuo. wrote the manuscript.

\section{Additional information}

Supplementary Information accompanies this paper at http://www.nature.com/ naturecommunications

Competing financial interests: The authors declare no competing financial interests.

Reprints and permission information is available online at http://npg.nature.com/ reprintsandpermissions/

How to cite this article: Jing, H. et al. Peptidyl-prolyl isomerization targets rice Aux/IAAs for proteasomal degradation during auxin signalling. Nat. Commun. 6:7395 doi: 10.1038/ncomms8395 (2015). 